\title{
A New Derivatization Strategy \\ for the Analysis of Phosphopeptides \\ by Precursor Ion Scanning in Positive \\ Ion Mode
}

\author{
Hanno Steen ${ }^{*}$ and Matthias Mann \\ Protein Interaction Laboratory, Center for Experimental Bioinformatics, Department of Biochemistry \\ and Molecular Biology, University of Southern Denmark, Odense, Denmark
}

\begin{abstract}
Although numerous strategies have been devised to analyze protein phosphorylation, an abundant intracellular protein modification, there is still a need for different methods for the analysis of this modification. A method to both detect and localize the phosphorylation within a protein/peptide is especially required. In this paper, a new strategy is described, which makes use of $\beta$-elimination/Michael addition reactions to introduce a functional group at the original site of phosphorylation, which gives rise to a dimethylamine-containing sulfenic acid derivative with a unique $\mathrm{m} / \mathrm{z}$ value. This enables the detection of the phosphorylated species within peptide mixtures by sensitive and specific precursor ion scanning in positive ion mode. Working under acidic conditions in positive ion mode has the added advantage that subsequent normal peptide sequencing for the exact localization can be performed. No other peptide derived fragment ion is observed at the $\mathrm{m} / \mathrm{z}$ value of the sulfenic acid derivative formed, thus specific precursor ion experiments can also be carried out on instruments with low fragment ion resolution and lends itself to LC-MS/MS approaches when skimmer fragmentation routines or triple quadrupole mass spectrometers are used. (J Am Soc Mass Spectrom 2002, 13, 996-1003) (c) 2002 American Society for Mass Spectrometry
\end{abstract}

A pproximately $30 \%$ of all mammalian proteins are phosphorylated at any given time and about $5 \%$ of most eukaroytic genomes encode for protein kinases and phosphatases, i.e., enzymes involved in protein phosphorylation and dephosphorylation [1]. This makes phosphorylation one of the most important intracellular protein modifications warranting the need for robust, fast, and sensitive methods for the analysis of protein phosphorylation, which includes both detection of the phosphorylated species and exact localization of the corresponding phosphorylation site. Apart from comprehensive LC-MS/MS analysis where phosphorylation sites are often found as a "side effect", two particular strategies out of a number of different mass spectrometry-based approaches for phosphorylation analysis are widely applied. One approach makes use of selective purification of the phosphorylated species using immobilized metal ion affinity chromatography (IMAC) followed by matrix-assisted laser desorption/ionization time-of-flight mass spectrometry

Published online June 27, 2002

Address reprint requests to Dr. M. Mann, Protein Interaction Laboratory, Department of Biochemistry and Molecular Biology, University of Southern Denmark, Campusvej 55, DK-5230 Odense M, Denmark. E-mail: mann@bmb.sdu.dk

${ }^{*}$ Current address: Department of Cell Biology, Harvard Medical School, 240 Longwood Avenue, Boston, MA 02115, USA.
(MALDI-TOF MS) analysis before and after phosphatase treatment. This enzyme treatment induces a characteristic mass shift of $-80 \mathrm{Da}$ for each phosphomoiety removed which allows the identification of the phosphopeptides but not the site of the modification [2-5]. The other commonly used strategy utilizes the characteristic $\mathrm{PO}_{3}^{-}$fragment ion at $\mathrm{m} / \mathrm{z}-79$ to identify phosphorylated species within a peptide mixture by precursor ion scanning [6-8]. Both strategies have proven to be very sensitive and specific for the detection of phosphorylated species. However, the exact localization of the phosphorylated amino acid residue in the peptide sequence is not simple since in the former case the phosphoamino acid residue is converted into its unmodified counterpart and in the latter case the detection is performed under alkaline conditions in negative ion mode, whereas sensitive peptide sequencing by MS/MS requires acidic conditions for positive ion mode experiments. This means that sample splitting or rebuffering is necessary, both of which compromise sensitivity and throughput.

The problem of identifying the phosphorylated species in peptide mixtures is further complicated by the mass spectrometric properties of the phosphomoiety itself, caused presumably by factors such as lower ionization efficiency and ionization suppression because of the highly acidic phosphomoiety. To circum- 
vent these problems, $\beta$-elimination/Michael addition of phosphopeptides have been devised. This strategy, using ethanethiol as the nucleophile for derivatization, was originally introduced by Meyer and co-workers in 1986 in order to overcome the problems associated with sequencing of phosphopeptides by Edman degradation [9]. $\beta$-Elimination-based approaches have the advantage that various analytical strategies become possible: Resing et al. removed the phosphomoiety without further Michael addition of a nucleophile so that the phosphoserine residues were converted into dehydroalanine residues which allowed the localization of the former phosphorylation site by subsequent MS/MS experiments. Thus, any phosphomoiety-related problems are avoided [10]. Jaffe et al. adopted a strategy for the mass spectrometric analysis of protein phosphorylation similar to the original work by Meyer et al. by using ethanethiol as nucleophile. However, isomeric methionine and ethylcysteine residues cannot be differentiated using mass spectrometry [11]. Weckwerth et al. combined this approach with stable isotope labeling using deuterated ethanethiol, thus enabling relative quantification of protein phosphorylation [12]. Even though the derivatized phosphopeptides show better ionization efficiencies than their phosphorylated counterparts, the problem of identifying the formerly phosphorylated species within complex sample mixtures persists in the above approaches as no characteristic signature is provided. To circumvent this shortcoming, Oda et al. introduced a strategy whereby the phosphomoiety was replaced by a biotin moiety, which allowed the selective avidin-based affinity purification [13]. Goshe et al. adopted this strategy and combined it in a preliminary study with stable isotope labeling for relative quantification of phosphorylation states [14]. Both approaches, however, suffer from problems such as inefficient elution from the streptavidin beads and low derivatization efficiency in the case of multiple phosphorylation in close vicinity.

Another approach to identify the phosphorylated species within a complex peptide mixture such as unseparated protein digests was recently published by Molloy and Andrews. The phosphopeptides were selectively labeled by $\beta$-elimination/Michael addition using two different alkanethiols as nucleophiles which differ by one methylene group. Hence, the ion signal of the formerly phosphorylated species is split in two, spaced by $14 \mathrm{Da}$, which allows the identification of the former phosphopeptides [15]. The disadvantages of this approach are that (1) it requires that the signals are clearly visible as such, i.e., signals hidden in the noise or with very low signal-to-noise ratios can be problematic, (2) the sensitivity is reduced as the signal is spread over more species, which is even more pronounced if multiple phosphorylation is observed.

In this paper we describe the combination of $\beta$-elimination/Michael addition derivatization and positive ion mode precursor ion scanning for the selective detection of peptides containing alkylphosphates, en- abling subsequent peptide sequencing. This complements the detection of tyrosine-phosphorylated species by positive ion mode precursor ion experiments which is possible without any derivatization by using the immonium ion of phosphotyrosine as reporter ion [16, 17]. The devised strategy introduces a strongly basic nucleophile which (1) improves the effective ionization of formerly phosphorylated species under acidic spray conditions and (2) gives rise to a highly abundant and characteristic fragment cation which can be detected by positive ion mode precursor ion scanning. Precursor ion scanning enables the sensitive detection of phosphopeptides in unseparated peptide mixtures such as protein digest even if the ion signals are hidden in the chemical noise. Since the identification of the formerly phosphorylated species is performed in acidic solution in positive ion mode, sequencing can follow immediately after the detection, i.e., prior knowledge of the protein is not required such that this method should also be applicable to proteins not known to be present and also to novel proteins.

\section{Materials and Methods}

\section{Materials}

Chemicals were obtained from Sigma-Aldrich (Vallensbaek Strand, Denmark). High purity solvents for mass spectrometric analysis were purchased from Labscan (Dublin, Ireland). The peptide APESL(pS)YDAR was custom made by Sigma-Genosys (Pampisford, UK). Trypsin (sequencing grade) and elastase were purchased from Roche-Diagnostics (Hvidovre, Denmark).

\section{Elastase in-Solution Digest of Ovalbumin}

Ovalbumin was dissolved in $5 \mathrm{mM} \mathrm{NH} \mathrm{NCO}_{3}$ at a concentration of $5 \mu \mathrm{M}$. Elastase was added [enzyme to protein ratio: 1:20 (w:w)] and the solution was incubated for $10 \mathrm{~h}$ at $37^{\circ} \mathrm{C}$.

\section{Purification of Phosphorylated Calmodulin}

The calmodulin in vitro phosphorylation mixture (kindly provided by Dr. Barbara Guerra, Department of Biochemistry and Molecular Biology, University of Southern Denmark, Denmark) was separated by SDSPAGE (4-12\% NuPage; Novex, San Diego, CA) and visualized by colloidal Coomassie Blue staining (Colloidal Blue Staining Kit, Novex) according to the manufacturer's instructions.

\section{$\beta$-Elimination/Michael Addition Using 2- Dimethylaminoethanethiol as Nucleophile}

The synthetic peptide $(1 \mathrm{nmol})$ was dissolved in $17.6 \mu \mathrm{l}$ of $0.54 \mathrm{M}$ 2-dimethylaminoethanethiol in $17 \%$ ethanol. $4.8 \mu \mathrm{l}$ of $5 \mathrm{M} \mathrm{NaOH}$ was added and the mixture was incubated at $56{ }^{\circ} \mathrm{C}$ for $60 \mathrm{~min}$. The reaction was 
quenched by adding $1 \mu \mathrm{l}$ formic acid. The solution was evaporated to dryness in a speedvac, redissolved in 100 $\mu \mathrm{l} 5 \%$ formic acid and a fraction was purified over a POROS R2 (Perseptive, Framingham, MA) micro-column prepared in GELoader tips (Eppendorf, Hamburg, Germany) [18, 19].

Elastase digest of ovalbumin $(20 \mathrm{pmol})$ or tryptic digest of calmodulin was treated for 5 min with 3\% $\mathrm{H}_{2} \mathrm{O}_{2}$. This mixture was subjected to solvent evaporation in a vacuum centrifuge. The peptide mixture was redissolved in $2 \mu \mathrm{l}$ of $0.54 \mathrm{M}$ 2-dimethylaminoethanethiol in 17\% ethanol: $\mathrm{H}_{2} \mathrm{O}: 5 \mathrm{M} \mathrm{NaOH}$ (4:1:1) and incubated at $56^{\circ} \mathrm{C}$ for $60 \mathrm{~min}$. The reaction was quenched by adding $100 \mu \mathrm{l}$ of $5 \%$ formic acid prior to loading onto a POROS R2 micro-column for extensive washing [18, 19].

\section{In-Gel Derivatization and Digestion of Calmodulin}

The gel band of interest was excised and reduced in-gel as described previously [20] and incubated with 3\% $\mathrm{H}_{2} \mathrm{O}_{2}$. After extensive washing, the gel plugs were shrunk with acetronitrile and reswollen in $40 \mu \mathrm{l}$ of 0.54 M 2-dimethylamino-ethanethiol in $17 \%$ ethanol: $\mathrm{H}_{2} \mathrm{O}: 5$ $\mathrm{M} \mathrm{NaOH}$ (4:1:1). After incubation at $56^{\circ} \mathrm{C}$ for $60 \mathrm{~min}$ the supernatant was removed and the gel plugs extensively washed with $5 \mathrm{mM} \mathrm{NH} \mathrm{HCO}_{3}$ and acetonitrile. The derivatized calmodulin was in-gel digested according to published procedures [20] and subjected to mass spectrometric analysis.

\section{Mass Spectrometry}

All experiments were performed on a QSTAR Pulsar quadrupole time-of-flight mass spectrometer (AB/MDS Sciex, Toronto, Canada), equipped with a nanoelectrospray ion source (Protana Engineering A/S, Odense, Denmark).

Precursor ion scans were acquired with a dwell time of $50 \mathrm{~ms}$ at a step size of $0.5 \mathrm{Da}$ and with the $\mathrm{Q}_{2}$-pulsing function turned on. Nitrogen was used as the collision gas at a recorded pressure of $5.3 \times 10^{-5}$ torr. The collision energy was ramped over the $\mathrm{m} / \mathrm{z}$ range proportional to one twentieth of the $\mathrm{m} / \mathrm{z}$ value of the precursor ion. Optimal collision energies may need to be determined empirically for different instruments.

The samples were desalted and concentrated on a micro-column (see above) and were eluted with $60 \%$ methanol in 5\% formic acid directly into nanoelectrospray needles (Protana Engineering).

\section{Results and Discussion}

A good nucleophile for the $\beta$-elimination/Michael addition derivatization of phosphopeptides with subsequent detection of the derivatized species by precursor ion scanning has to fulfill several requirements. (1) As the limit of detection for particular precursor ion experiments is directly related to the signal intensity of the characteristic reporter ion, the fragment ion used for this purpose should be highly abundant in product ion spectra under the conditions applied for precursor ion scanning. (2) The reporter ion must have a unique mass to ensure specificity of the precursor ion experiments. Thus, the modified amino acid residue has to give rise to a characteristic fragment ion with an $\mathrm{m} / \mathrm{z}$ value where no other peptide derived fragment ions, such as immonium ions or a-, b-, and y-ions including the loss of ammonia and water, respectively, are expected. (3) The formation of the characteristic fragment ion should not interfere with the formation of sequence-revealing fragments, i.e., a fragmentation inducing "mobile" proton has to remain on the protonated peptide after formation of the reporter ion [21,22]. Otherwise sequencing and exact localization of the former site of phosphorylation can be hindered [23].

The chosen derivatization strategy that fulfills all requirements mentioned above is shown in Figure 1. The $\beta$-elimination/Michael addition is performed using the nucleophile 2-dimethylaminoethanethiol and the generated thioether is converted into the corresponding sulfoxide derivative upon treatment with hydrogen peroxide. The generated sulfoxide undergoes facile gas phase $\beta$-elimination as we recently described for peptides containing oxidized alkylated cysteine residues [24]. We reasoned that this gas phase elimination reaction should generate an intense protonated sulfenic acid derivative at $\mathrm{m} / \mathrm{z} 122.06$ (see Figure 1), thereby satisfying requirement (1). Calculation of all possible peptide derived fragment ions $(\mathrm{a}-, \mathrm{b}-$, and $\mathrm{y}$-ions including the losses of ammonia and water) shows that no fragment ion is expected at $\mathrm{m} / \mathrm{z} 122.06$, i.e., it is unique and therefore also fulfills requirement (2). 2-dimethylaminoethanethiol used for the derivatization is highly basic due to the tertiary amino group and does not interfere with peptide fragmentation as it does not remove the "mobile proton" from the protonated peptide species during the gas phase $\beta$-elimination. Thus, the requirement (3) (see above) is fulfilled by 2-dimethylaminoethanethiol used as reagent for the derivatization.

In order to validate the chosen strategy, the phosphopeptide APESL(pS)YDAR was treated with strong base in the presence of 2-dimethylaminoethanethiol. The mass spectra of the serine-phosphorylated peptide before and after derivatization are shown in Figure 2a and $b$. The introduction of a new highly basic side chain, replacing the highly acidic phosphate group, results in the shift of the main charge state from $2+$ before the derivatization reaction to $3+$ afterwards. The fragment ion spectrum of the doubly protonated peptide at $m / z 606.26$ is shown in Figure 2c. The major ion signals in the product ion spectrum are the sulfenic acid derivative at $\mathrm{m} / \mathrm{z} 122.06$ and the complimentary fragment at $\mathrm{m} / \mathrm{z}$ 1090.51, corresponding to the peptide APESL(dhA)YDAR (dhA: dehydroalanine; product of the gas phase $\beta$-elimination reaction after solution phase derivatization reaction). This peptide(-fragment) fragments after the labile loss of the sulfenic acid 


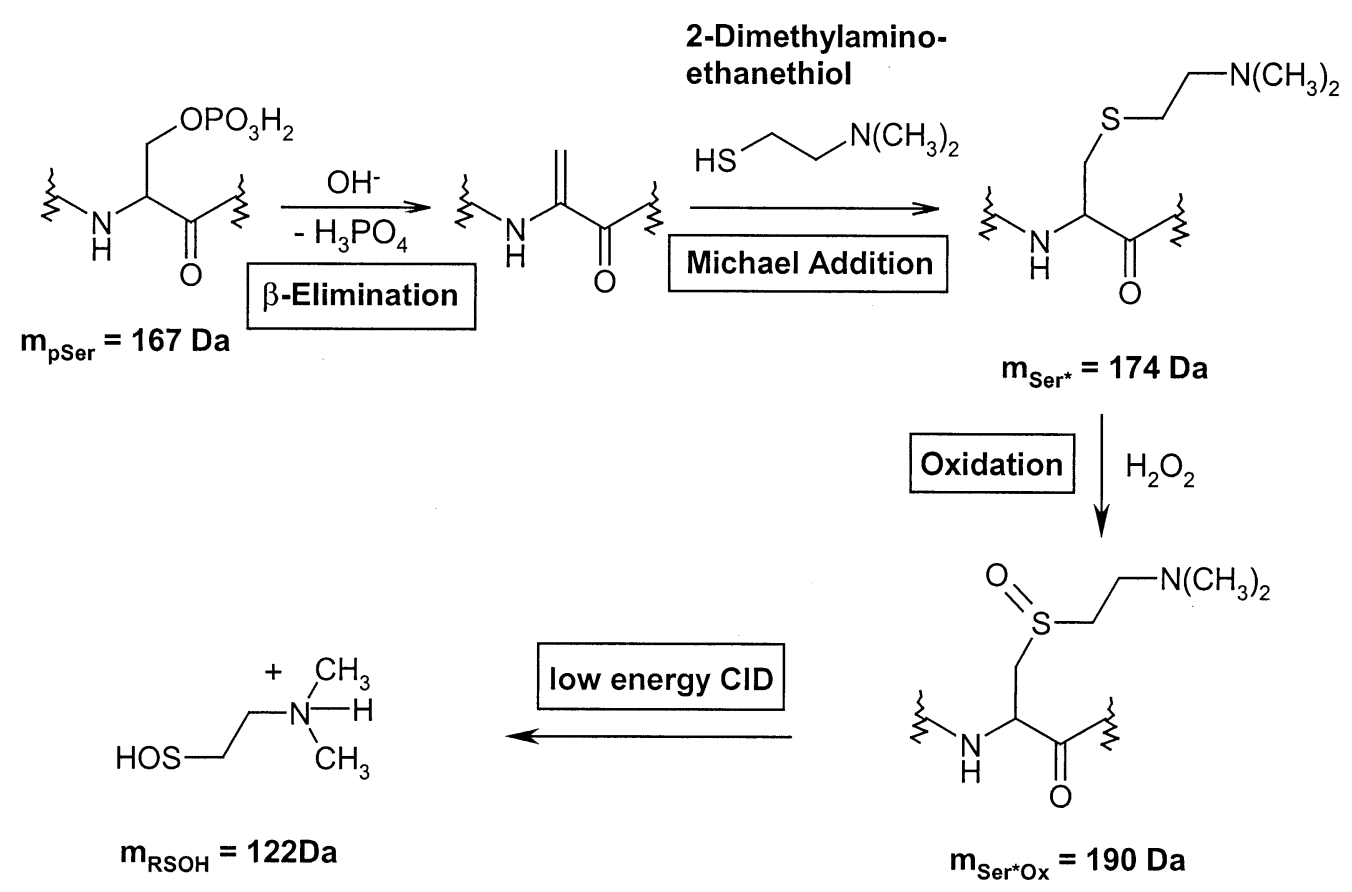

Figure 1. Derivatization strategy for serine-phosphorylated peptides. Serine-phosphorylated peptides undergo $\beta$-elimination upon treatment with strong bases. The resulting Michael system reacts with the nucleophilic thiol group of 2-dimethylaminoethanethiol which introduces an additional highly basic functional group at the former phosphorylation site. Controlled oxidation of the thioether to the sulfoxide is accomplished by short incubation with $3 \% \mathrm{H}_{2} \mathrm{O}_{2}$. The generated 2-dimethylaminoethanesulfoxide derivative gives rise to a characteristic fragment ion (sulfenic acid derivative) at $\mathrm{m} / \mathrm{z}$ 122.06 upon low energy CID.

derivative similar to ordinary tryptic peptides, such that the complete y-ion series (after the gas phase elimination of the sulfenic acid derivative) is apparent as the main fragment ion series. Y-type fragment ions that include the sulfoxide moiety, i.e., one which is 122 Da heavier than the observed one, are hardly visible in the product ion spectrum (ratio $>50: 1$; see Figure 2e; marked with triangles) indicating the low activation barrier for this gas phase elimination reaction. The formerly serine-phosphorylated species can be identified based on the mass difference of 69 Da corresponding to dehydroalanine resulting from the $\beta$-elimination of the sulfoxide derivative. Formerly threonine-phosphorylated species generate 2-aminodehydrobutyric acid derivatives upon derivatization and gas-phase $\beta$-elimination with an mass difference of $83 \mathrm{Da}$. Therefore standard peptide sequencing can be employed with two "new" amino acid in both cases: Dehydroalanine (69 Da) and 2-aminodehydrobutyric acid (83 Da).

For further testing of the applicability of this method to peptide mixtures such as protein digests, this derivatization procedure was applied to an in-solution digest of ovalbumin using elastase as the proteolytic enzyme. All disulfide bridges were reduced with DTT and then treated with $3 \% \mathrm{H}_{2} \mathrm{O}_{2}$ before digestion thus converting thioethers (methionine residues) into sulfoxides and thiols (reduced cysteine residues) into sulfonic acid derivatives. These precautions have to be taken in order to minimize the risk of false positives since unmodified methionine and cysteine residues undergo the same elimination reaction as the phosphoserine residues. Subsequently the protein was digested with elastase and the resulting peptide mixture was dried in the speedvac and derivatized in the same way as the synthetic peptide described above. A complex peptide mixture was obtained due to the low specificity of the protease (see Figure 3a). The following precursors of $(\mathrm{m} / \mathrm{z} 122)$ experiment significantly reduced the complexity of the digest allowing the identification of the formerly serine-phosphorylated peptides (see Figure $3 \mathrm{~b}$, marked with asterisks). MS/MS experiments of the peptides giving rise to the main ion signals in the precursor ion spectrum confirmed Ser-68 and Ser-344 as the two serine phosphorylation sites in ovalbumin. The MS/MS spectrum of the peptide at $\mathrm{m} / \mathrm{z} 594.9$ corresponding to the peptide RFDKLPGFGD $\left(\mathrm{S}^{*}\right)$ IEAQ is shown in Figure 3c. The mass difference of $69 \mathrm{Da}$ between two adjacent $b$-fragment ions allowed the unambiguous localization of the former phosphorylation site (see Figure 3d).

The in-solution derivatization experiments showed near-complete conversion. Using the same protocol for in-gel derivatization of gel-separated proteins, the conversion yield did not exceed $60 \%$ presumable because of reduced accessibility of the phosphorylation sites in-gel. However, since cleanup of the sample is greatly facilitated when the reaction is performed in-gel, the 

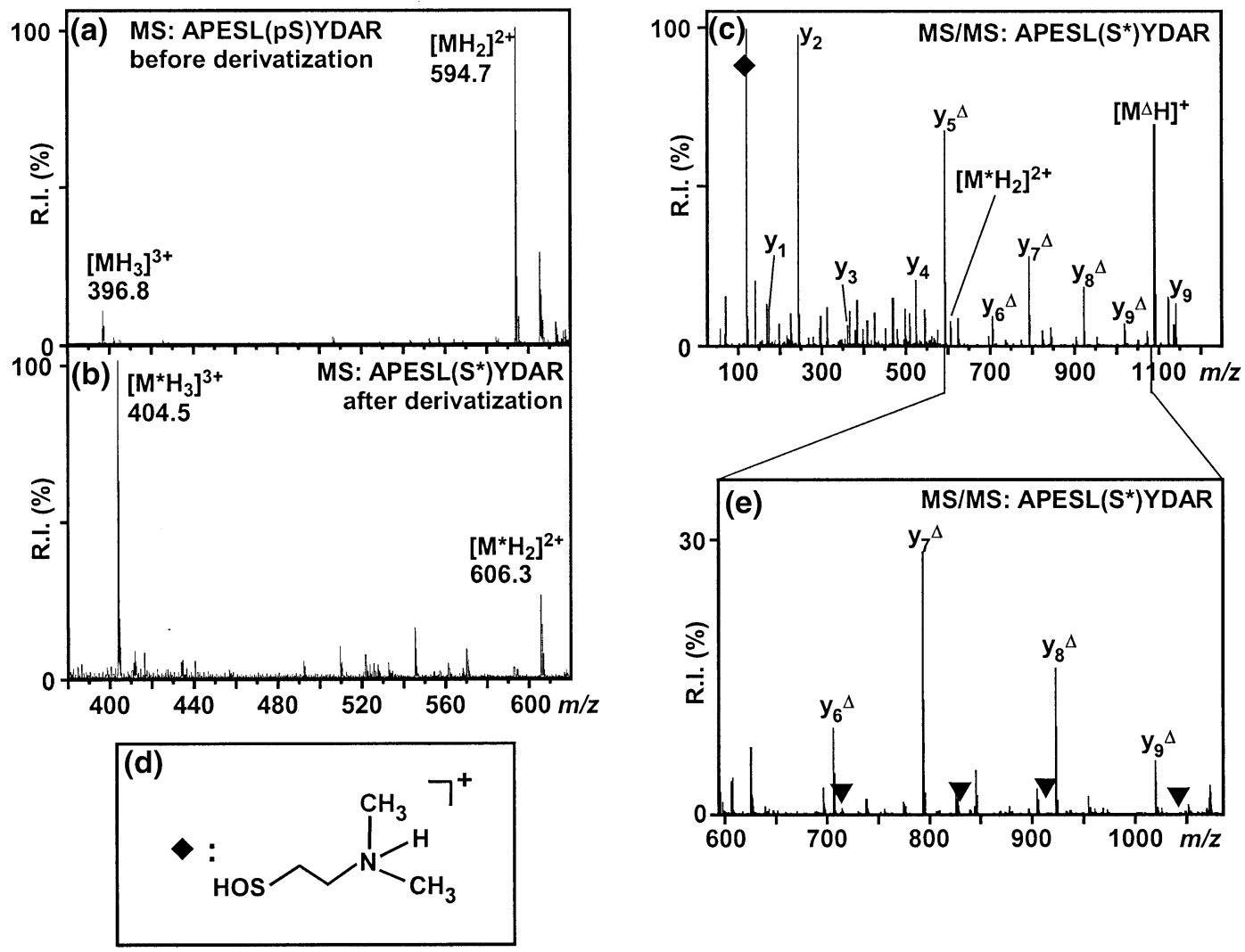

Figure 2. Mass spectrometric analysis of the derivatization reaction for serine-phosphorylated peptides. (a) Mass spectrum of the synthetic peptide APESL(pS)YDAR before the derivatization reaction. The base peak is the doubly protonated species. (b) Mass spectrum of the peptide APESLS*YDAR after $\beta$-elimination/Michael addition/oxidation derivatization shown in Figure 1. The newly introduced highly basic amino group results in the triply protonated species being the base peak. (c) Product ion spectrum of the doubly charged derivatization product. The former phosphoserine residue was clearly identified as dehydroalanine (dhA) based on a residue mass of $69 \mathrm{Da}$. The characteristic protonated sulfenic acid derivative is marked with a filled square (the structure is shown in (d). All major fragment ions underwent peptide backbone cleavage and gas phase $\beta$-elimination (dhA-containing fragments are labeled with $\Delta$ ). Hardly any fragments which only underwent peptide backbone cleavages without $\beta$-elimination were observable [marked with filled triangles; see (e)].

complete protein was derivatized in-gel in the following experiment of this preliminary study.

After the successful proof of this concept, the derivatization method was applied to calmodulin (CaM), which was phosphorylated in vitro by casein kinase 2 in order to substantiate this concept. The starting amount of CaM was approximately $6 \mathrm{pmol}$, and neither the degree nor the exact site of phosphorylation under the applied phosphorylation conditions was known. CaM was separated from the other proteins present in the reaction mixture by SDS-PAGE and visualized using colloidal Coomassie Blue staining. The derivatization reaction with 2-dimethylaminoethanethiol was performed as described above, after reduction of the disulfide bonds and oxidation of the thioethers and thiols to minimize side reactions (see above). The strong alkaline conditions resulted in partial hydrolysis of the gel matrix, and therefore a significantly increased volume of the gel plugs, such that for the subsequent tryptic in-gel digestion using established protocols [20] a large volume of trypsin solution was required for reswelling the gel plugs. The mass spectrum of the protein digest is shown in Figure 4a and displays mainly trypsin autolysis peaks. The subsequent precursors of $(\mathrm{m} / \mathrm{z}$ $122.06 \pm 0.02)$ experiment is displayed in Figure $4 \mathrm{~b}$. Three prominent precursor ion signals at $m / z 465,518$, and 620 were observable. The ion signal at $\mathrm{m} / \mathrm{z} 465$ corresponded to a quadruply charged precursor $\mathrm{m} / \mathrm{z}$ 465.48 that could be distinguished from chemical noise because of the $\mathrm{m} / \mathrm{z} 0.25$ spacing of the peptide isotope peaks (see Figure 4c). The other two ion signals were attributable to triply charged precursors at $\mathrm{m} / \mathrm{z} 518.26$ and 620.31 with a signal to noise ratio of 2-3 (see Figure $4 \mathrm{~d})$. The product ion spectrum of the peptide at $\mathrm{m} / \mathrm{z}$ 518.26 revealed the presence of the modified tryptic autolysis peptide LQGIVSWGSGCAQK (207-220) (data not shown). Thorough interpretation of the data revealed the presence of an undesired disulfide containing side product resulting from a reaction of 2-dimethylaminoethanethiol with the cysteine residue Cys-217 which is involved in a disulfide bridge in the active form of trypsin. Even though thorough washing of the 

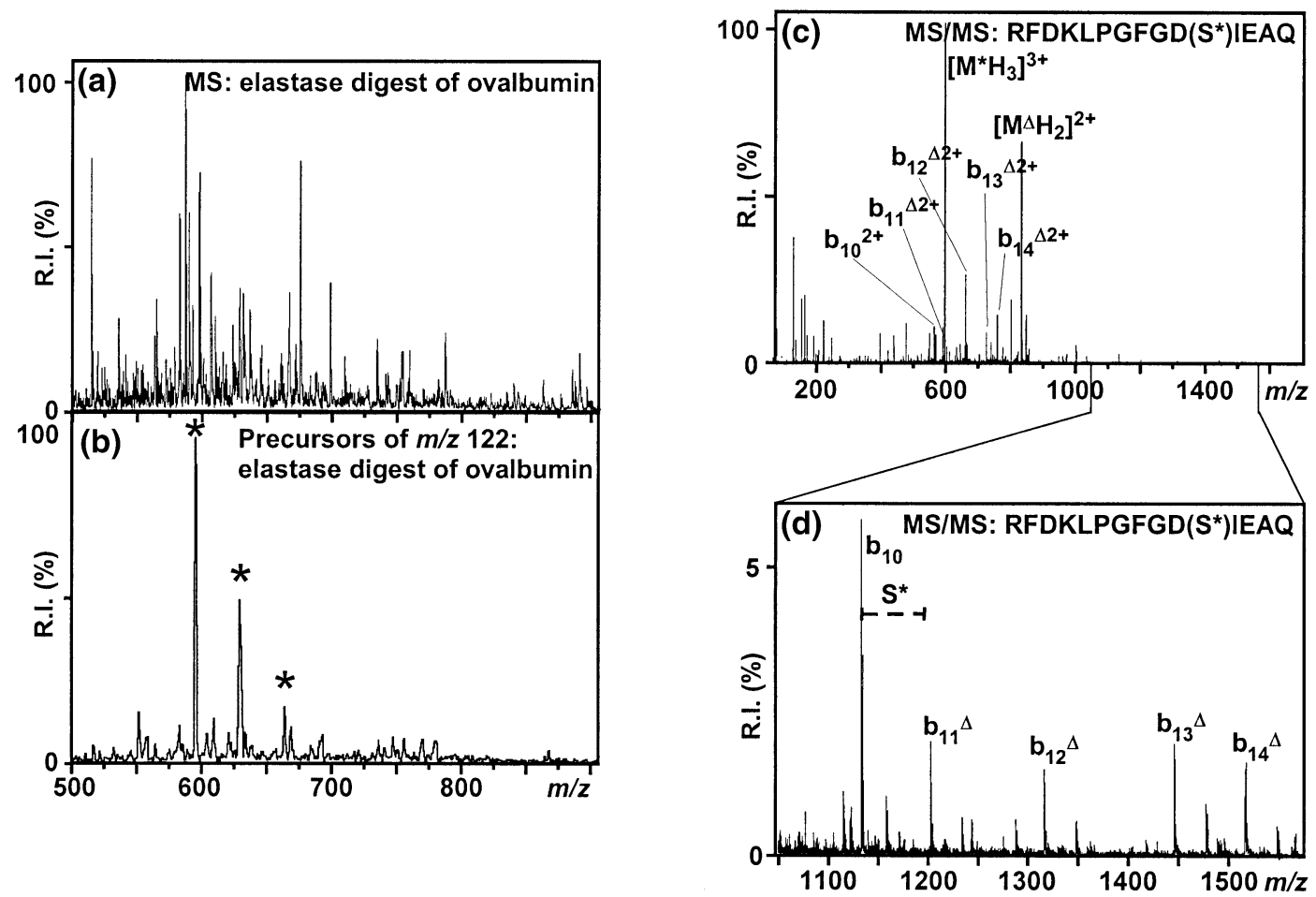

Figure 3. Mass spectrometric analysis of a derivatized in-solution elastase digest of ovalbumin. Ovalbumin was digested in solution with elastase prior to the derivatization procedure shown in Figure 1. (a) Mass spectrum of the derivatized elastase digest. (b) Precursor of $(\mathrm{m} / \mathrm{z} 122.06 \pm 0.02)$ experiment. Three major ion signals are observable (marked with asterisks), corresponding to the triply charged peptides RFDKLPGFGD(S*)IEAQ $\left(\mathrm{m} / \mathrm{z}\right.$ 594.9; $\mathrm{S}^{*}$ denotes the former phosphoserine residue), VRFDKLPGFGD( $\left.\mathrm{S}^{*}\right)$ IEAQ $\left(\mathrm{m} / \mathrm{z}\right.$ 627.9), and HAEINEAGREVVG( $\left.\mathrm{S}^{*}\right)$ AEAGV $(\mathrm{m} / \mathrm{z}$ 666.7) as shown by further MS/MS experiments. (c) Product ion spectrum of the triply charged precursor at $\mathrm{m} / \mathrm{z}$ 594.9 allowed the confirmation of Ser-68 within the peptide RFDKLPGFGD(S*)IEAQ as being originally phosphorylated. Fragment ions which underwent gas phase $\beta$-elimination, i.e., which contained dehydroalanine instead of the sulfoxide moiety, are labeled with $\Delta$. (d) Expansion of the higher $\mathrm{m} / \mathrm{z}$ range of the product ion spectrum displaying the singly charged b-ion series which allows the unambiguous localization of the former phosphoserine residue.

gel pieces was performed, the residual amounts of 2-dimethylaminoethanethiol were sufficient to cause these problems. A similar problem is frequently encountered for residual iodoacetamide and trypsin in ordinary identification projects of gel-separated proteins. This indicates that complete removal of the reagents has to be ensured if the derivatization reaction is performed before the digest.

The product ion spectra of the other two species at $\mathrm{m} / \mathrm{z} 465.48$ and 620.31 were related to the same peptide species but in different protonation states. It corresponded to the triply and quadruply charged tryptic peptide from CaM comprising the amino acid residues 91-105 [VFDKDGNGYI(S*)AAELR; $\mathrm{S}^{*}$ denotes the former phosphoserine residue]. The product ion spectrum of the triply charged ion species at $\mathrm{m} / \mathrm{z} 620.31$ is shown in Figure 4e. This spectrum exhibits the same features as the one described for the synthetic peptide (see above): The major fragment ions are attributable to the sulfenic acid derivative at $\mathrm{m} / \mathrm{z} 122$ and its complimentary ion marked with $\left[\mathrm{M}^{\Delta} \mathrm{H}_{2}\right]^{2+}$. Apart from these two fragment ions, the complete y-type fragment ion series after the elimination of the sulfenic acid is observable. The data allowed unambiguous localization of the formerly phosphorylated serine residue, based on the 69 Da mass difference of the $y_{6}$ and $y_{5}$ fragment ions. This Ser-101 is within a well-documented casein kinase 2 phosphorylation site (S/T-X-X-E/D versus S-A-A-E in $\mathrm{CaM}$ ) of calmodulin. This residue has been shown to be phosphorylated in vivo [25] as well as in vitro by casein kinase 2. In the latter case phosphorylation yields of Ser-101 of up to $25 \%$ had been described [26].

\section{Conclusions and Perspective}

The approach described in this paper utilizes a $\beta$-elimination/Michael addition strategy to introduce a side chain with favorable properties such as the formation of a characteristic fragment ion, which allows the use of precursor ion experiments for the selective detection of the formerly phosphorylated species. Proof of principle has been provided using synthetic peptides and protein digests in-solution as well as in-gel, showing the feasi- 

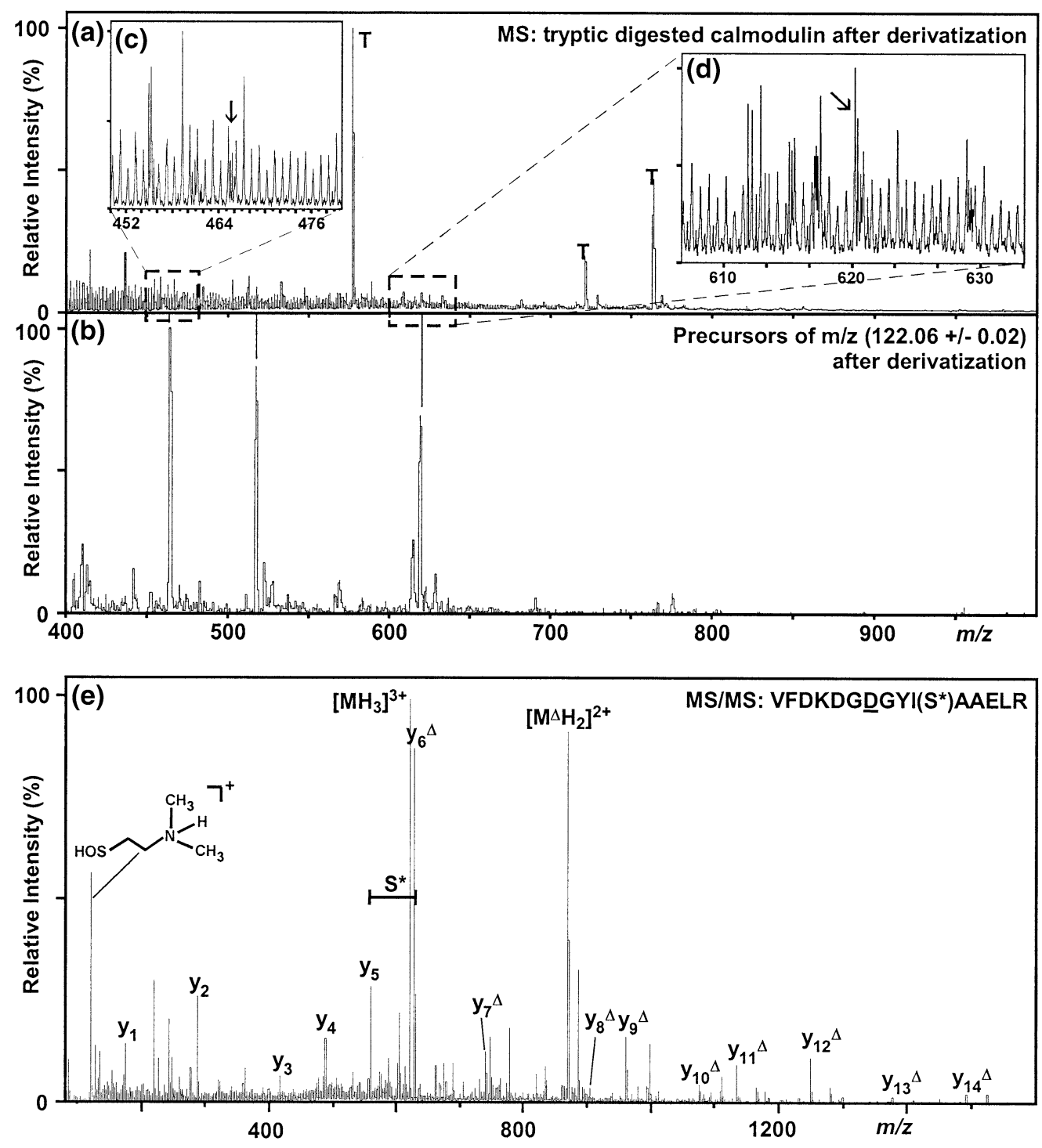

Figure 4. Mass spectrometric analysis of a tryptic digest of derivatized phosphorylated calmodulin. Calmodulin was phosphorylated in vitro with casein kinase 2 in the presence of polylysine and subsequently purified by SDS-PAGE. The gel band of interest was excised and subjected to the derivatization shown in Figure 1 prior to in-gel tryptic digestion. (a) Mass spectrum of the tryptic digest. The major ion signals were attributed to trypsin autolysis products and are marked with T. (b) Selective precursor of $(\mathrm{m} / z 122.06 \pm 0.02)$ experiment. Three major ion signals are observable. (c) Expansion of the corresponding $\mathrm{m} / \mathrm{z}$ ranges of the mass spectrum of the tryptic digest revealed the presence of a low abundance quadruply (c) and triply (d) charged precursor. Product ion spectrum of the triply charged precursor at $m / z 620.31$ allowed the localization of Ser-101 within the tryptic peptide $\mathrm{T}_{91-105}$ VFDKDGNGYI( $\left.\mathrm{S}^{*}\right)$ AAELR ( $\mathrm{S}^{*}$ denotes the former phosphoserine residue) as being initially phosphorylated. Fragment ions which underwent gas phase $\beta$-elimination, i.e., which contained dehydroalanine instead of the sulfoxide moiety, are labeled with $\Delta$. The underlined aspartic acid residue is a deamidation product.

bility of this approach even for in vitro phosphorylated, gel-separated protein sample.

Precursor ion scanning has the advantage of superior detection limits compared to normal MS modes. This allows the detection of phosphorylated species even when the corresponding ion signal has a signal-to-noise ratio of below one, i.e., is not observable. The introduced functional group carries a highly basic tertiary amino group generally resulting in an increase of the charge state by one. The generation of the characteristic fragment ion does not remove any "labile" proton and the subsequent fragmentation of the peptide for the exact localization of the former site of phosphorylation is not hampered. Another benefit of the characteristic fragment ion formed upon low energy CID is its mass of $122 \mathrm{Da}$. No interfering peptide-derived fragment ions are found at this $m / z$ value, i.e., precursor ion experiments can also be performed on a low-resolution instrument and do not require high-resolution, high-accuracy quadrupole TOF mass spectrometers as in the case of 
positive ion mode precursor ion experiments for the analysis of tyrosine-phosphorylated peptides [16, 17].

Even though the derivatization protocol used for this study allows for the easy differentiation between serine- and threonine-phosphorylation, further studies will focus on optimizing the yields of the derivatization of the latter kind of phosphorylation and the in-gel derivatization, and minimizing the unwanted side reactions of the $\beta$-elimination reaction in general. Other future directions comprise the combination of this method with LC-MS/MS experiments for the "on the fly"detection of phosphorylated species, and the use of an isotopically labeled derivatization agent for relative quantification of phosphorylation states.

\section{Acknowledgments}

The authors wish to thank Dr. B. Guerra (Department of Biochemistry and Molecular Biology, University of Southern Denmark) for of the provision of the in vitro phosphorylated calmodulin sample. They also acknowledge Drs. J. Jebanathirajah and J. Rappsilber for critically reading the manuscript and Dr. T. J. D. Jørgensen and O. Hørning for their fruitful discussions about the oxidation of thioethers (all from the Department of Biochemistry and Molecular Biology, University of Southern Denmark). Work in the authors' laboratory is funded by a grant from the Danish National Research Foundation to the Center for Experimental Bioinformatics (CEBI).

\section{References}

1. Hunter, T. The Croonian Lecture 1997. The Phosphorylation of Proteins on Tyrosine: Its Role in Cell Growth and Disease. Phil. Trans. R. Soc. Lond. B 1998, 353, 583-605.

2. Andersson, L.; Porath, J. Isolation of Phosphoproteins by Metal (Fe3+) Affinity Chromatography. Anal. Biochem. 1986, 154, 250-254.

3. Nuwaysir, L. M.; Stults, J. T. Electrospray Ionization Mass Spectrometry of Phosphopeptides Isolated by on-Line Immobilized Metal-Ion Affinity Chromatography. J. Am. Soc. Mass Spectrom. 1993, 4, 662-669.

4. Wang, K. Y.; Liao, P.-C.; Allison, J.; Gage, D. A.; Andrews, P. C.; Lubman, D. M.; Hanash, S. M.; Strahler, J. R. Phorbol 12-Myristate 13Acetate-Induced Phosphorylation of Op18 in Jurkat T Cells. J. Biol. Chem. 1993, 268, 14269-14277.

5. Stensballe, A.; Andersen, S.; Jensen, O. N. Characterization of Phosphoproteins from Electrophoretic Gels by Nano-Scale $\mathrm{Fe}(\mathrm{III})$ Affinity Chromatography with Off-Line Mass Spectrometry Analysis. Proteomics 2001, 1, 207-222.

6. Carr, S. A.; Huddleston, M. J.; Annan, R. S. Selective Detection and Sequencing of Phosphopeptides at the Femtomole Level by Mass Spectrometry. Anal. Biochem. 1996, 239, 180-192.

7. Wilm, M.; Neubauer, G.; Mann, M. Parent Ion Scans of Unseparated Peptide Mixtures. Anal. Chem. 1996, 68, 527-533.

8. Annan, R. S.; Huddleston, M. J.; Verma, R.; Deshaies, R. J.; Carr, S. A. A Multidimensional Electrospray MS-Based Approach to Phosphopeptide Mapping. Anal. Chem. 2001, 73, 393-404.

9. Meyer, H.; Hoffmann-Posorske, E.; Korte, H.; Heilmeyer, L. J. Sequence Analysis of Phosphoserine-Containing Peptides. Modification for Picomolar Sensitivity. FEBS Lett. 1986, 204, 61-66.

10. Resing, K. A.; Johnson, R. S.; Walsh, K. A. Mass Spectrometric Analysis of 21 Phosphorylation Sites in the Internal Repeat of Rat Profilaggrin, Precursor of an Intermediate Filament Associated Protein. Biochem. 1995, 34, 9477-9487.
11. Jaffe, H.Veeranna;; Pant, H. C. Characterization of Serine and Threonine Phosphorylation Sites in Beta-Elimination/Ethanethiol Addition-Modified Proteins by Electrospray Tandem Mass Spectrometry and Database Searching. Biochem. 1998, 37, 16211-16224.

12. Weckwerth, W.; Willmitzer, L.; Fiehn, O. Comparative Quantification and Identification of Phosphoproteins Using Stable Isotope Labeling and Liquid Chromatography/Mass Spectrometry. Rapid Commun. Mass Spectrom. 2000, 14, 1677-1681.

13. Oda, Y.; Nagasu, T.; Chait, B. T. Enrichment Analysis of Phosphorylated Proteins as a Tool for Probing the Phosphoproteome. Nat. Biotechnol. 2001, 19, 379-382.

14. Goshe, M. B.; Conrads, T. P.; Panisko, E. A.; Angell, N. H.; Veenstra, T. D.; Smith, R. D. Phosphoprotein Isotope-Coded Affinity Tag Approach for Isolating and Quantitating Phosphopeptides in Proteome-Wide Analyses. Anal. Chem. 2001, 73, 2578-2586.

15. Molloy, M. P.; Andrews, P. C. Phosphopeptide Derivatization Signatures to Identify Serine and Threonine Phosphorylated Peptides by Mass Spectrometry. Anal. Chem. 2001, 73, 53875394.

16. Steen, H.; Küster, B.; Fernandez, M.; Pandey, A.; Mann, M. Sensitive Detection of Tyrosine Phosphorylated Peptides by Precursor Ion Scanning Quadrupole TOF Mass Spectrometry in Positive Ion Mode. Anal. Chem. 2001, 73, 1440-1448.

17. Steen, H.; Küster, B.; Mann, M. Quadrupole Time-of-Flight Versus Triple-Quadrupole Mass Spectrometry for the Determination of Phosphopeptides by Precursor Ion Scanning. J. Mass Spectrom. 2001, 36, 782-790.

18. Gobom, J.; Nordhoff, E.; Mirgorodskaya, E.; Ekman, R.; Roepstorff, P. Sample Purification and Preparation Technique Based on Nano-Scale Reversed-Phase Columns for the Sensitive Analysis of Complex Peptide Mixtures by Matrix-Assisted Laser Desorption/Ionization Mass Spectrometry. J. Mass Spectrom. 1999, 34, 105-116.

19. Neubauer, G.; Mann, M. Mapping of Phosphorylation Sites of Gel-Isolated Proteins by Nanoelectrospray Tandem Mass Spectrometry: Potentials and Limitations. Anal. Chem. 1999, 71, 235-242.

20. Shevchenko, A.; Wilm, M.; Vorm, O.; Mann, M. Mass Spectrometric Sequencing of Proteins from Silver-Stained Polyacrylamide Gels. Anal. Chem. 1996, 68, 850-858.

21. Wysocki, V. H.; Tsaprailis, G.; Smith, L. L.; Breci, L. A. Mobile and Localized Protons: A Framework for Understanding Peptide Dissociation. J. Mass Spectrom. 2000, 35, 1399-1406.

22. Polce, M. J.; Ren, D.; Wesdemiotis, C. Dissociation of the Peptide Bond in Protonated Peptides. J. Mass Spectrom. 2000, 35, 1391-1398.

23. Cramer, R.; Corless, S. The Nature of Collision-Induced Dissociation Processes of Doubly Protonated Peptides: Comparative Study for the Future Use of Matrix-Assisted Laser Desorption/Ionization on a Hybrid Quadrupole Time-ofFlight Mass Spectrometer in Proteomics. J. Mass Spectrom. 2001, 15, 2058-2066.

24. Steen, H.; Mann, M. Similarity between Condensed Phase and Gas Phase Chemistry: Fragmentation of Peptides Containing Oxidized Cysteine Residues and Its Implications for Proteomics. J. Am. Soc. Mass Spectrom. 2001, 12, 228-232.

25. Quadroni, M.; James, P.; Carafoli, E. Isolation of Phosphorylated Calmodulin from Rat Liver and Identification of the in Vivo Phosphorylation Sites. J. Biol. Chem. 1994, 269, 1611616122.

26. Sacks, D. B.; Davis, H. W.; Williams, J. P.; Sheehan, E. L.; Garcia, J. G.; McDonald, J. M. Phosphorylation by Casein Kinase Ii Alters the Biological Activity of Calmodulin. Biochem. J. 1992, 283, 21-24. 